\title{
Für internationale Vergleichsmessungen optimierter Drehmoment - Transferaufnehmer TN
}

\author{
Dr.- Ing. André Schäfer \\ Anschrift: \\ Hottinger Baldwin Messtechnik GmbH (HBM), \\ Darmstadt, Germany, \\ Tel.-Nr.: +49-6151-803224; \\ E-Mail-Adresse: andre.schaefer@hbm
}

\section{Zusammenfassung}

Drehmoment-Referenzaufnehmer, welche als Transferstandards für die Rückführung der Messgröße Drehmoment dienen, werden in der Regel auf der Basis von Dehnungsmessstreifen aufgebaut. Um von den Vorteilen eines komplett monolithischen Designs in metrologischen Anwendungen profitieren zu können, muss dieser Drehmomentsensor in Schaftform ausgeführt werden. Schaftsensoren wiederum können nur "offen" ausgeführt werden, anders als bei der üblicheren Ausführung als Flansch, wo die Dehnungsmessstreifen (DMS) hermetisch gekapselt werden können. Die Ausführungsform Schaft ist also eine besondere Herausforderung, da Folien-DMS sind ohne hermetische Kapselung recht stark von den Umgebungsbedingungen abhängig sind und so hierauf besonderes Augenmerk gelegt werden muss.

Da Drehmoment- Transferstandards für Vergleiche zwischen internationalen Metrologie-Laboren gedacht sind, ist deren Verwendbarkeit auch unter veränderten Bedingungen von großer Bedeutung. Die Gesamtzeit der Messungen aller Labore inkl. der Transferzeit soll kurz sein, die Ergebnisse sollen eine bestimmte Messunsicherheit bestätigen. Daher wurden Auswirkungen bestimmter Anwendungsbedingungen, und zwar ebenso für die benötigte Hochpräzisionselektronik, eingehend untersucht.

Keywords: Drehmoment-Referenzaufnehmer, Dehungsmessstreifen, DMS, Wheatstone-Brücke, Präzisionsmessverstärker, Präzisionsbrückenverstärker

\section{Einführung}

Seit dem Jahre 1958 offeriert HBM Drehmomentmesswellen. Die im Zusammenhang mit dieser Messgröße stehenden Geschäfte wuchsen vor allem in den letzten drei Jahrzehnten recht schnell. Im Jahre 1990 erlangte die am Stammhaus in Darmstadt aufgebaute $25 \mathrm{kNm}$ Bezugsnormalmesseinrichtung sogar die Rolle eines nationalen Normals. Diese Anlage war damals auf der Basis eines Schneidenlagers aufgebaut worden. Heute verwendet man vor allem Luftlager, die noch einmal deutlich reibungsärmer sind.

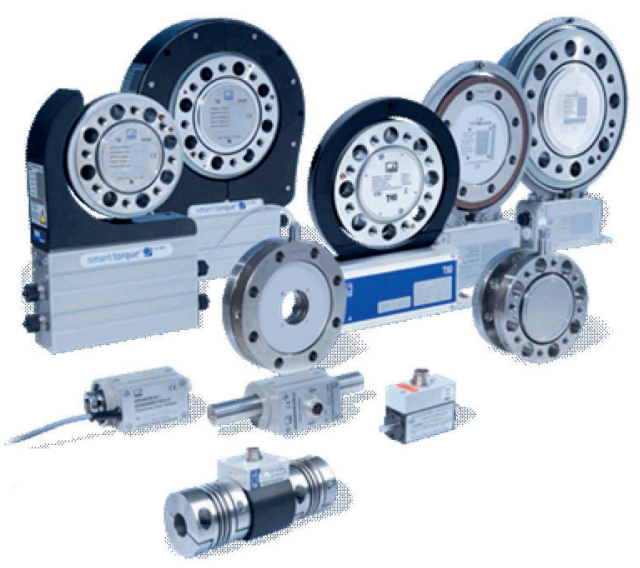

Abbildung 1: Wichtige Baureihen des HBM Drehmomentaufnehmer-Programms 
Um die Forderungen der Automobilindustrie zu meistern, müssen Drehmomente gemäß den angegebenen und weiter reduzierten Unsicherheiten dargestellt und weitergegeben werden. Daher müssen Messketten, bestehend aus Referenzaufnehmern und Präzisionsinstrumenten, die z. B. für die Rückführung von Drehmomentschlüsseln in diesen Drehmomentlaboratorien vorgesehen sind, hohe Anforderungen erfüllen. Zugleich muss die Präzisionsmesskette portabel sein, um als stabiler "Travel Standards" für Vergleiche zwischen Labors verwendet werden zu können, wobei auf dem Weg vom Labor zum Labor eine bestimmte Zeit erforderlich sein wird, um sich an das Klima im Labor anzupassen und die Messung schließlich starten zu können.

Neben der oft gut dokumentierten Temperatur muss hier auch die Luftfeuchtigkeit sorgfältig, als mögliche Quelle von Messunsicherheiten, betrachtet werden. Hygroskopische Substanzen reichern sich mit Wasser an. Diese verdient vor allem im DMSAufnehmerbau großes Augenmerk, da bei "offenen" DMS-Konstruktionen deren Signal durch ein ggf. hygroskopisches Verhalten des DMS-Trägers beeinflusst wird.

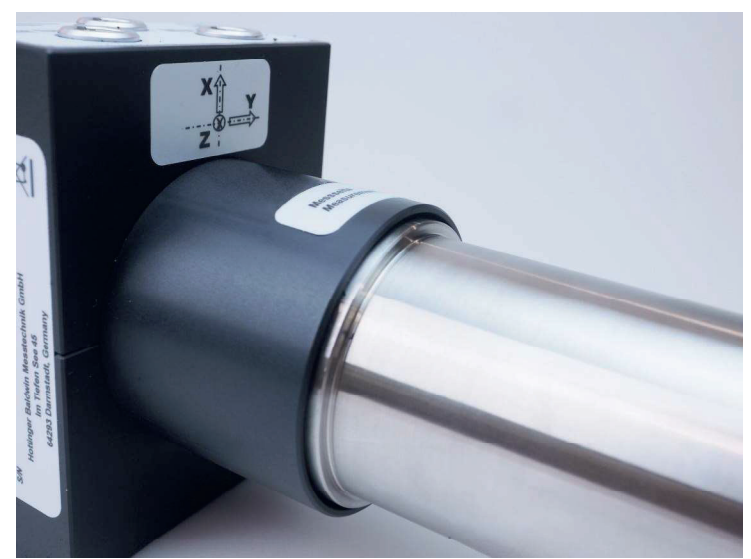

Abbildung 2:

Bei Schaftaufnehmern erforderlicher Ringspalt: Um einen Kraftnebenschluss zu verhindern, muss der Rotor vollständig vom Rotor getrennt sein. Durch den erforderlichen Ringspalt kann Umgebungsluft in die DMS eindringen.

Verdeutlichen kann man sich dies, indem man sich einen Schwamm vorstellt, der das Wasser aus der Umgebung aufsaugt, und der sich daher vergrößert. Vier DMS sind in der Wheatstone-Brücke verschaltet, aber auch die externe Beschaltung, z.B. zur Temperaturkompensation, kann dies geschehen. Wenn ein solcher DrehmomentReferenzaufnehmer auf DMS-Basis und in "offener Bauart" unterschiedlichen Feuchtigkeitswerten ausgesetzt ist, zeigt er eine Reaktion sowohl in der Empfindlichkeit als auch im Nullsignal.

Die Wirkung auf das Nullsignal ist zwar oft deutlich höher als die Empfindlichkeitsänderung, jedoch kann dies weitestgehend durch Tarieren vor dem Messvorgang kompensiert werden. Der Einfluss auf die Empfindlichkeit, also Sensitivität, schlägt jedoch voll durch auf das Ergebnis der Labore und eine Minimierung dieses ist für die Verwendbarkeit in Vergleichen zwischen Labors unabdingbar [1]; [2].

\section{Der neue Referenzdrehmomentaufnehmer}

Die Schaft-Grundform ist bereits seit vielen Jahren Basis für die Referenzdrehmomentaufnehmer-Serie "TN“ von HBM. Er wurde bereits weitestgehend auf den Einsatz als "Transfer standard“" in Kalibrierlaboratorien gemäß DIN 51309 z.B. in DAkkS-akkreditierten Stellen ausgelegt.

Von den Messbereichen her wird weiterhin für Nenndrehmomente 100, 200, $500 \mathrm{Nm}$ und 1, 2, 5, 10 und $20 \mathrm{kNm}$ angeboten. Spezielles Design, aber auch der Lieferumfang des Aufnehmers wurden hingegen weiter optimiert.

Nun wird auch die bisher oft gefragte Biegemomentoption Bestandteil des Lieferumfangs. Das bedeutet, dass der neue TN neben dem Drehmomentmesskanal nun standardmäßig mit zwei BiegemomentMesskanälen (X-/Y-Richtung) ausgestattet ist, um mögliche Lage-Abweichungen zu detektieren.

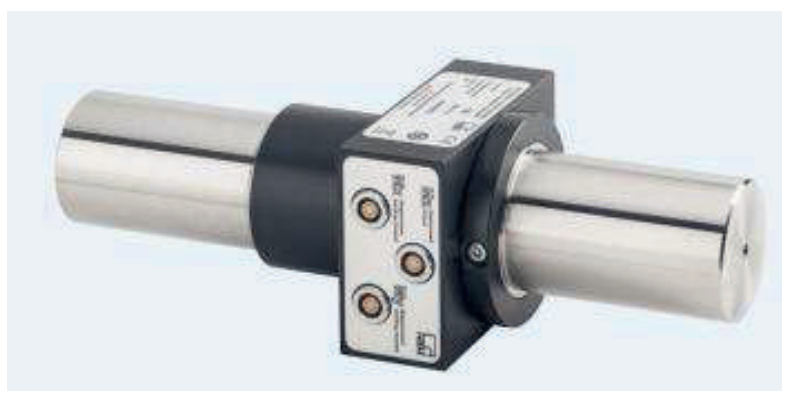

Abbildung 3: TN-Referenz-drehmomentaufnehmer in seiner neuen Ausprägung. Auf der Frontseite: neben dem Drehmomentmesskanal nun standardmäßig zwei Biegemoment-Messkanäle (X-/Y-Richtung) 
Zusätzlich ist nun ein Tragekoffer im Standardlieferumfang enthalten, welcher mit einer speziellen Dichtlippe ausgestattet ist, die einen guten mechanischen Schutz, aber eben auch eine gute Feuchtigkeitsisolierung bietet. Aufgrund des erweiterten StandardLieferumfangs ist zukünftig nur noch eine Ausprägung pro Messbereich erforderlich, was auch die Lieferzeit verkürzt.
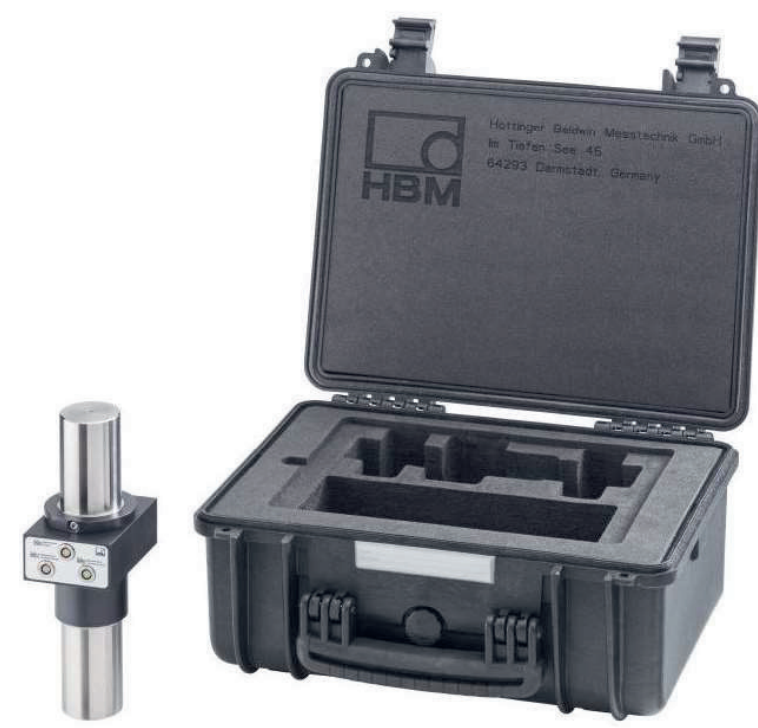

Abbildung 4:

Neuer, kompakter Transportkoffer mit spezieller, umlaufender Dichtlippe

\section{Untersuchungen zur Empfindlichkeit gegenüber Feuchtigkeit}

Die Feuchtigkeitsempfindlichkeit ist nicht nur ein Thema für den Drehmomentaufnehmer, sondern für die gesamte hochpräzise Messkette. Das dieser Tatsache ist trotz der Klimatisierung der Labore der Nationalen Metrologie-Institute (NMI) große Bedeutung beizumessen ist, zeigen Berichte von „Key Comparisions" als auch speziell durchgeführte Untersuchungen [3]; [4].

Die generelle Einsicht, dass es angesichts deutlicher Fehlerbilder infolge Luftfeuchtigkeit sinnvoll ist, auf klimasichere Komponenten der Referenzkette, d.h. sowohl Aufnehmer als auch Elektronik hinzuarbeiten, ist seit langem klar [5]; [6].
Zunehmend stellte sich heraus, dass mit immer kleiner werdendem Messunsicherheitsbudget der Einfluss der Luftfeuchtigkeit nicht mehr vernachlässigbar ist. In Bezug auf den Aufnehmer haben sich die NMls bereits seit einigen Jahren mit dem Problem befasst [7]; [8]. Schließlich wurden auch für die Präzisionsinstrumente entsprechende Untersuchungen angeregt [9]; [10]; [11].

Die für die Anwendung „internationale Vergleichs-messung" so wichtige Unempfindlichkeit gegenüber Feuchtigkeit aus der Umgebungsluft konnte nun, im Ergebnis umfangreicher Untersuchungen erstmalig in der DMS-Messtechnik für die Messkette quantifiziert werden.

Daher konnten hier folgende Angaben zum Einfluss der relativen Feuchte von TN ermittelt werden:

\begin{tabular}{|l|l|l|}
\hline Gerätefamilie & $\begin{array}{l}\text { In den Aus- } \\
\text { prägungen } \\
\text { (Bestell- } \\
\text { nummer) }\end{array}$ & $\begin{array}{l}\text { Feuchte- } \\
\text { empfindlichk } \\
\text { eit - } \\
\text { typische } \\
\text { Werte }\end{array}$ \\
\hline $\begin{array}{l}\text { Transfer- } \\
\text { normal TN } \\
\text { (neue Aus- } \\
\text { führung } \\
2019 / 2020)\end{array}$ & $\begin{array}{l}1-\mathrm{TN} / 100 \mathrm{Nm} \\
1-\mathrm{TN} / 200 \mathrm{Nm} / 500 \mathrm{Nm} \\
1-\mathrm{TN} / 1 \mathrm{kNm} \\
\mathrm{ppm} / \% \mathrm{rH}\end{array}$ \\
& $\begin{array}{l}\text { Typ. }<10 \\
1-\mathrm{TN} / 2 \mathrm{kNm}\end{array}$ & \\
& $1-\mathrm{TN} / 5 \mathrm{kNm}$ & \\
& $1-\mathrm{TN} / 20 \mathrm{kNm}$ & \\
\hline
\end{tabular}

\section{Schlussfolgerungen}

Obwohl bereits vielfach bewährt, konnte der Drehmoment-Transferaufnehmer TN in seiner Eignung für internationale Vergleichsmessungen noch einmal deutlich verbessert werden.

Für die gegebenen Konstruktionsoptionen bieten die Verbesserungen am Sensor in Verbindung mit dem neuen Tragekoffer nicht nur ausreichenden mechanischen Schutz bei trotzdem hoher Portabilität, sondern eben auch 
eine höhere Immunität gegenüber verschiedensten Umgebungsbedingungen.

Dabei ermöglicht eine Verringerung der Feuchtigkeitsempfindlichkeit aufgrund der Verwendung eines deutlich weniger hygroskopischen DMS-Trägermaterials es auch die erforderliche Zeit, welche für eine ausreichende "Klimaanpassung" vor Messbeginn in jedem Labor erforderlich ist, entsprechend zu verkürzen.

Die Motivation hierfür ist, dass mit ihm vor allem primäre Anwendungen bedient werden sollen und daher kleinste Messunsicherheiten erforderlich sind. Dazu muss ein deutlich größerer Aufwand getrieben werden.

Je geringer das Messunsicherheitsbudget ist, desto mehr Parameter stellen sich für dessen Einhaltung als relevant heraus. Dafür betreffen die Maßnahmen hin zu einer noch besseren Feuchtigkeitsunempfindlichkeit den Aufbau des Aufnehmers selbst wie auch den neuen Tragekoffer, zumal dieser zusätzlich auch einen besseren mechanischen Schutz gegen unterschiedlichste Umgebungsbedingungen bietet.

Im Allgemeinen ermöglicht eine Verringerung der Feuchtigkeitsempfindlichkeit/ -aufnahme durch eine bessere Verpackung und die Verwendung weniger hygroskopischer DMSWerkstoffe außerdem eine Verkürzung der oben erwähnten Zeit für die „Akklimatisation“ vor dem Start der Messung.

Für Ringvergleiche zwischen Labors und die kleinste Messunsicherheit kombiniert man am besten TN-Aufnehmer mit einem DMP41-T6 in einer Präzisionsmesskette [12].

\section{Literaturnachweis}

[1] Röske, D.; Mauersberger, D. On the stability of measuring devices for torque key comparisons, IMEKO XVIII World Congress \& IV Brazilian Congress of Metrology, September 2006, Rio de Janeiro, Brazil.

[2] Brüge, A. Influence of humitidy on torque estimation methods for calibration laboratories; XX IMEKO World Congr. 2012, Busan, Rep. of Korea

[3] Röske, D., Key comparisons in the field of torque measurement, 19th IMEKO TC3 on Force, Mass and Torque: "Theory and Application in Laboratories and Industries", 2005, Cairo, Egypt.
[4] Röske, D. Final report on the torque key comparison CCM.T-K1: Measurand torque: 100 $\mathrm{N} \cdot \mathrm{m}, 500 \mathrm{~N} \cdot \mathrm{m}, 1000 \mathrm{~N} \cdot \mathrm{m}$, Metrologia, 46 (2009), Tech. Suppl., 07006.

[5] Ring, K. Klimasicherheit von elektronischen Baugruppen (engl. transl.: Climate safety of electronic assemblies), ZVE Fraunhofer Institut für Zuverlässigkeit \& Mikrointegration, Berlin, 2007

[6] Käs, G. Qualität und Zuverlässigkeit elektronischer Bauelemente und Systeme, R. Oldenburg publishing, Vienna, 1983, ISBN3486-2735-5

[7] Brüge, A. Simplified measurements of the humidity coefficient of torque transducers in calibration laboratories, ACTA IMEKO June 2014, Volume 3, Number 2, 32 - 38, www.imeko.org

[8] Khaled, K.M.; Röske, D. at al. Humidity and temperature effects on torque transducers, bridge calibration unit and amplifiers,

Measurement 74 (2015) p. 31-42, Elsevier B.V. Publishing house, N.Y.; U.S.A.

[9] Drung, D. at al ; Improvements in the field of traceable high-accuracy measurement and generation of small electrical currents, Presentation of Helmholtz award winners, PTB WG 2.61, Bad Honnef, Germany, May 2018

[10] Schäfer, A. The Ultra-Precision Instrument DMP41 -First Experiences \& Appropriate Filter settings, TC3 Conference, 2014, Cape Town, South Africa

[11] Schäfer, A. DMP41-A new Chapter of UltraPrecision Instrument for Strain Gauge Transducers XX IMEKO World Congr. 2012, Busan, Rep. of Korea

[12] Schäfer, A. Notwendigkeiten und Gesetzmäßigkeiten beim Aufbau von Präzisionsmessketten auf Basis von Dehnungsmessstreifen. Tagung Innovation Messtechnik, Linz, Österreich, Mai 2019 